\title{
Propagación y crecimiento inicial del abarco (Cariniana pyriformis Miers), utilizando semillas silvestres
}

\section{Propagation and initial growth of abarco (Cariniana pyriformis Miers), using wild seed}

\author{
Propagação e crescimento inicial do abarco
} (Cariniana pyriformis Miers), utilizando sementes silvestres

\author{
Harlenson Pinilla Cespedes ${ }^{1}$, Henry Hernan Medina Arroyo² ${ }^{2}$, Jhon Jerley Torres Torres ${ }^{3}$, \\ Estivinson Córdoba Urrutia ${ }^{4}$, Juan Carlos Córdoba Moreno ${ }^{5}$, \\ Yosuar Mosquera Ampudia ${ }^{6}$ \& Melida Martínez Guardia ${ }^{7}$

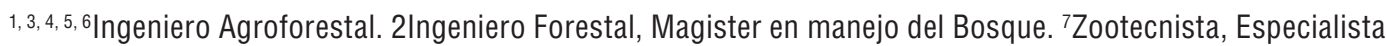 \\ en Informática Educativa, Magister en Ciencia de Reproducción Animal, Doctora en Ciencia Animal \\ 1, 2, 3, 4, 5, 6,7 Grupo de Investigación Ciencia Animal y Recursos Agroforestales. \\ Universidad Tecnológica del Chocó “Diego Luis Córdoba”. Quibdó, Colombia \\ 1harlenson9@hotmail.com, ${ }^{2}$ hehemear@gmail.com, 3jhonjerleytorres@gmail.com, ${ }^{4}$ scordobau@gmail.com, \\ ${ }^{5}$ carlos.0791@hotmail.com, ${ }^{6}$ themaravillos010@hotmail.com, ${ }^{7}$ melidamaguar@yahoo.es
}

\section{Resumen}

Se realizó la propagación de la especie forestal Abarco Cariniana Pyriformis Miers, con el objetivo de analizar su comportamiento en vivero utilizando semillas botánicas no mejoradas. Se utilizó un diseño completamente aleatorio con arreglo factorial compuesto por 10 factores por condición lumínica -luz y sombra- y 45 unidades experimentales. Se utilizaron diferentes combinaciones de sustratos orgánicos. Las semillas fueron sometidas a un tratamiento pre-germinativo en presencia de un testigo. Las variables evaluadas fueron porcentaje de germinación, crecimiento en altura y diámetro y porcentaje de supervivencia. La especie comenzó la germinación 13 días después de la siembra y se extendió hasta el día 27. Los resultados del proceso de germinación fueron de $60 \%$ y $40 \%$ a plena exposición solar y bajo malla poli-sombra, respectivamente. EI crecimiento en altura de las plántulas muestra al factor CF con el mejor valor $28,94 \mathrm{~cm}$, bajo condiciones de luz y el factor $E F$, con $25,56 \mathrm{~cm}$ a plena exposición de sombra. Mientras el incremento en diámetro los factores DF y EF registraron valores de $1,04 \mathrm{~cm}$ y $0,84 \mathrm{~cm}$, bajo las condiciones sol y sombra respetivamente. Las semillas de esta especie son no-fotoblásticas, es decir que para su germinación es indiferente la presencia o ausencia de luz. Las plántulas ubicadas en la condición ambiental sol registraron mejores valores promedios en las variables diámetro y altura. Por tanto, esta condición ejerce efecto positivo para el crecimiento inicial de las plántulas de abarco C. pyriformis.

Palabras claves: germinación, pre-germinativos, semillas, sustrato, tratamiento. 


\section{Abstract}

The propagation of the forest species abarco Cariniana Pyriformis Miers was carried out with the objective of analyzing its behavior in nursery using botanical seeds not improved. A completely randomized design with a factorial arrangement composed of 10 factors per light condition -light and shade- And 45 experimental units. Different combinations of organic substrates were used. The seeds used were pre-germinated in the presence of a control. The variables evaluated were percentage of germination, growth in height and diameter and percentage of survival. The species began germination 13 days after sowing and extended to day 27. The results of the germination process were $60 \%$ and $40 \%$ at full sun exposure and under polyshade mesh respectively. The height growth of the seedlings shows the CF factor with the best value $28.94 \mathrm{~cm}$, under light conditions and EF factor, with $25.56 \mathrm{~cm}$ at full shadow exposure. While the increase in diameter DF and EF factors registered values of 1.04 and $0.84 \mathrm{~cm}$ under sun and shade conditions respectively. The seeds of this species are non-photoblastic, meaning that the presence or absence of light is indifferent to its germination. The seedlings located in the environmental sun condition recorded better average values in the variables diameter and height. Therefore, this condition exerts a positive effect on the initial growth of abarco C. pyriformis seedlings.

Key-words: germination, pre-germinative, seed, substrate, treatment.

\section{Resumo}

Realizou-se a propagação da espécie florestal Cariniana Pyriformis Miers, com objetivo de analisar seu comportamento em viveiro utilizando-se sementes botânicas não melhoradas. Foi feito um delineamento de blocos ao acaso com 10 fatores por condição luminosa -luz e sombra- e 45 unidades experimentais. Utilizaram-se diferentes misturas de substratos orgânicos. As sementes foram submetidas a um tratamento pre-germinação na presença de um controle. As variáveis avaliadas foram percentual de germinação, crescimento em altura de diâmetro e percentual de supervivência. A espécie começou a germinação 13 dias após do plantio e foi até o dia 27. Os resultados do processo de germinação foram 60 $\%$ e $40 \%$ exposição solar plena e baixo sombrite, respectivamente. $\mathrm{O}$ crescimento em altura das plântulas mostram o fator CF como o melhor fator com valor de 28,94 cm, em condições de luz e o fator EF, com $25,56 \mathrm{~cm}$ com total exposição na sombra. Enquanto isso, o incremento no diâmetro dos fatores DF e EF mostraram valores de $1,04 \mathrm{~cm}$ e $0,84 \mathrm{~cm}$, em condições de sol e sombra respectivamente. As sementes desta espécie são não-fotoblásticas, ou seja, que para a germinação não faz diferença a presença ou ausência de luz. As plântulas localizadas na condição ambiental de sol registraram melhores valores médios para as variáveis diâmetro e altura. Isso quer dizer que essa condição tem efeito positivo no crescimento inicial das plântulas de abarco C. pyriformis.

Palavras-chave: germinação, pre-germinativos, semente, substrato, tratamento.

\section{Introducción}

La biodiversidad en Colombia en diversas manifestaciones, ha permitido que, ocupe el segundo lugar entre los doce primeros países del mundo en diversidad biológica y endemismos, esto obedece a que, aporta el $10 \%$ de biodiversidad a nivel mundial (Bryant, 1997); la cual, está representada en gran parte, por cinco eco regiones como son: Llanuras del Caribe, Amazonía, Orinoquia, la Región Andina y Chocó Biogeográfico (Hernández, Hurtado, Ortiz
\& Walschburger, 1992), siendo este último, uno de los lugares con mayor biodiversidad del planeta (Díaz \& Gast, 2009).

La biodiversidad está siendo amenazada por actividades antrópicas como la deforestación, la apertura de tierras para la agricultura y ganadería, la construcción de obras civiles, la extracción minera y el aprovechamiento insostenible de productos y 
beneficios forestales (CORANTIOQUIA, 2007; Secretaria de Convenio sobre la Diversidad Biológica, 2009), lo cual ha ocasionado reducciones notables de especies vegetales de alto valor comercial, entre las que se mencionan al abarco Cariniana pyriformis Miers, lo anterior se debe, a la calidad y durabilidad de la madera que es muy apreciada tanto en el mercado nacional como internacional. Esta situación ha generado que en Colombia los registros indiquen, reducciones de hasta $80 \%$ de sus individuos (Farfán, 2012).

En el departamento del Chocó, el cual hace parte del Chocó biogeográfico, el abarco C. pyriformis es transformada en dos productos madera rolliza y aserrada, siendo esta ultima la más representativa, ya que de ella se obtienen subproductos como: bloques, tablas, trozas, tablones, vigas, postes y polines; lo cual ha originado su incorporación entre las 6 especies que representan el $50 \%$ de la madera extraída y comercializada en este departamento (Informe de Cobertura de Desarrollo Regional, 2012). Lo anterior, ha originado que la especie este catalogada como en peligro crítico (CR) a la extinción a nivel regional (Corporación Autónoma Regional para el desarrollo Sostenible del Chocó "CODECHOCO", 2010; Klinger, 2008), como a nivel nacional (Cárdenas \& Salinas, 2005; Galeano et al., 2007). Lo que es revalidado por la Unidad Internacional de Conservación Natural UICN (2012).

En coherencia con lo anterior, Klinger (2008) manifiesta que esta situación de la especie $C$. pyriformis y en general las selvas del Chocó exige a las autoridades ambientales ejercer control sobre las actividades que deterioran los ecosistemas boscosos y restringen su conservación y protección. Algunas estrategias se han adelantado al respecto, sobresaliendo entre estas la emprendida en el año 2010, por el Ministerio de Ambiente, Vivienda y Desarrollo Territorial "MAVDT" mediante la resolución $\mathrm{N}^{\circ} 383$ del 23 de febrero declara a él abarco C. pyriformis en peligro crítico de extinción (CR), otorgando veda total a la especie. Otra acción adoptada por el gobierno nacional fue la estructuración del establecimiento de un fondo de capital privado forestal, donde se pretendía tener más árboles sembrados, más empleo, mejores condiciones de vida para nuestros campesinos, menor presión en el bosque natural y mejores beneficios ambientales para las comunidades (FEDEMADERAS, 2012).

Sumado a esto, aunque se ha avanzado en estrategias para la propagación en vivero y conservación del abarco C. pyriformis con diferentes sustratos orgánicos (Arena, tierra de bosque, gallinaza, hojarasca, tierra de hormiga y cascara de arroz) y diferentes tratamientos pre germinativos precisamente inmersión en agua a diferentes horas (CONAFOR, 2010; CATIE, 2002; CORANTIOQUIA, 2007; Betancur \& Raigosa, 1973; Guerra \& Montoya, 2013; Mosquera et al., 2012 \& Pinilla, 2015), se debe agregar que la mayoría de estos estudios fueron realizados con semillas certificadas provenientes de la casa comercial Semicol. S.A.

Basado en lo anterior, el propósito de esta investigación fue evaluar el efecto de tratamientos pre germinativos y sustratos orgánicos en la germinación y el crecimiento inicial del abarco C. pyriformis, bajo dos condiciones de luminosidad -libre exposición solar y bajo sombra- utilizando semillas silvestres, semillas botánicas no mejoradas. Este recurso representa una importancia incalculable para la preservación de los bosques y las especies nativas en los diferentes ecosistemas (CORANTIOQUIA, 2007). Además de esto cobra una amplia validez para los campesinos y productores, ostentada en la consecución inmediata de las semillas sin necesidad de comprarlas en una casa comercial, estas semillas generalmente se pierden o se descomponen y en algunos casos son de consumo para los animales, por consiguiente se hace importante que los seres humanos asentados en los distintos ecosistemas boscosos le den otra utilidad a este recurso genético.

\section{Materiales y métodos}

\section{Área de estudio}

El estudio se realizó en el corregimiento de Salero, el cual, se encuentra ubicado al norte del municipio de Unión Panamericana, en el departamento del Chocó, Colombia, sobre las coordenadas $\mathrm{X}=1.080 .403$ 
y $Y=1.050 .096$, a una distancia de $3 \mathrm{~km}$ de la cabecera municipal de las Animas. La población de salero es de 480 habitantes aproximadamente, (IIAP, 2006), presenta una temperatura promedio anual mayor de $24^{\circ} \mathrm{C}$ y precipitación menor de $8000 \mathrm{~mm}$ anuales, según el sistema de clasificación de Holdridge (1987) pertenece a la zona de vida bosque pluvial tropical bp-T.

Material vegetal y sustratos. Se utilizaron semiIlas silvestres de la especie en estudio, provenientes de los bosques del municipio de Ríosucio en el departamento del Chocó, Colombia. Los sustratos orgánicos utilizados en esta investigación fueron obtenidos del área de estudio con la colaboración de la comunidad. Este material estuvo compuesto por arena de rio, cascara de arroz, gallinaza, tierra negra y tierra de hormiga.

Construcción y adecuación del vivero. Se construyó un vivero de $256 \mathrm{~m}^{2}$, en el cual, se construyeron dos camas de germinación, una por condición lumínica (plástico transparente y malla poli-sombra al $85 \%$ ), con dimensiones de $1 \mathrm{~m}$ de ancho por $8 \mathrm{~m}$ de largo y $0.30 \mathrm{~m}$ de profundidad, la madera fue inmunizada con aceite quemado e inventrina para repeler el ataque de hormigas arrieras (Atta colombica).

Desinfección del sustrato orgánico. Los sustratos antes mencionados fueron desinfectados utilizando formol al $20 \%$ e inventrina. Se mezclaron 10 $\mathrm{ml}$ de formol y $10 \mathrm{ml}$ de Inventrina por cada $20 \mathrm{~L}$ de agua; esta sustancia se aplicó a los sustratos orgánicos hasta humedecerlo completamente. Al finalizar este procedimiento se cubrieron los sustratos con un plástico negro, el cual se destapaba cada 24 horas para remover y oxigenar el sustrato, a los cuatro días se dio por terminada la desinfección.

Tratamientos pre-germinativos. Los tratamientos pre germinativos a las semillas de C. pyriformis, fueron inmersión en agua ambiente durante 24 horas y el testigo, sin tratamiento pre -erminativo.

Factores utilizados en la investigación. La Tabla 1 presenta los factores que son el resultado de la combinación de sustratos orgánicos y tratamientos pre germinativo.

Tabla 1. Factores (Sustratos y tratamientos pre-germinativos).

B: Arena aluvial + Tierra negra 2:1

C: Tierra negra + Arena aluvial 2:1

D: Tierra de hormiga + Cascara de arroz 3:1

G: Inmersión de las semillas en agua a temperatura ambiente durante 24 horas

E: Tierra negra + Arena aluvial + Gallinaza 1:1:1

EF EG 
Siembra de las semillas de abarco Cariniana pyriformis. La siembra se efectuó en las dos (2) eras de germinación antes mencionadas, con representación de diez (10) factores y tres (3) repeticiones por cada factor. Se sembraron 15 semillas por cada repetición, 45 por factor, 450 por cada condición ambiental -bajo malla poli-sombra al $85 \%$ de opacidad y bajo plástico transparente-, para un total de 900 semillas entre las dos eras, la distancia de siembra fue de $10 \mathrm{~cm}$ entre semillas e hileras con profundidad de $2 \mathrm{~cm}$ debido al tamaño de la misma ya que esta tiene germinación epigea (CORANTIOQUIA, 2007).

Variables evaluadas. En esta investigación se evaluó la germinación, la cual fue registrada diariamente a través de la emergencia de los cotiledones del sustrato, una vez terminado este proceso se continuo con la medición de las variables crecimiento en diámetro y altura de la especie, hasta que alguna de las plantas alcanzaran la altura de trasplante a sitio definitivo. Las mediciones se efectuaron cada ocho días en un periodo de cuatro meses. Adicional a esto, se evaluó la supervivencia de las plántulas para cada factor.

Para evaluar la potencia germinativa y supervivencia se utilizaron las ecuaciones 1 y 2 :

\section{Potencia germinativa}

$(P G)=(S g / S s) \times 100 \%$

$\mathbf{S g}=$ semillas germinadas $y \mathrm{~S} s=$ semillas sembradas .

\section{Supervivencia}

$\% \mathbf{S V}=$ (número de plantas vivas / número de plantas muertas) ${ }^{*} 100$

Respecto a las actividades complementarias en la toma de datos en el vivero, se realizaron limpiezas en las eras de germinación, para contrarrestar la competencia a las plántulas de abarco C. pyriformis con otras especies consideradas arvenses. De igual forma, se realizaron riegos para el proceso de germinación y crecimiento inicial de las plántulas en las horas de la mañana y en la tarde en los días en que no se presentaban lluvias.

Diseño experimental. El experimento se estableció bajo un diseño completamente aleatorizado con arreglo factorial. Cada factor contó con tres repeticiones. La combinación de los dos factores principales -sustratos orgánicos y tratamientos pregerminativos- dio como resultado 10 factores, los cuales fueron sometidos a las dos condiciones lumínicas tal como se expresa en la Tabla 1.

Análisis estadístico. Los diferentes factores fueron comparados mediante un análisis de varianza (Alfa $0,5)$ y el Test de Tukey; usando el programa estadístico INFOSTAT (versión libre 2013), las variables evaluadas fueron crecimiento en diámetro y altura.

\section{Resultados}

La germinación de las semillas de C. pyriformis, inicio a los 13 días después de la fecha de siembra, donde se evidencio que, los cotiledones emergieron fuera del nivel del sustrato, presentando una germinación epigea. El proceso de germinación de las semillas de $C$. pyriformis, se extendió hasta el día 27 después de la fecha de siembra.

En el proceso de germinación se observó que las semillas sin tratamiento pre-germinativo, testigo, registro valores de $33,1 \%$, mientras que las semillas que fueron tratadas en inmersión en agua ambiente durante 24 horas registraron porcentajes de germinación de 29, 1\%.

Respecto a los factores, se observó que al evaluar los sustratos orgánicos con los tratamientos pregerminativos se evidencia un incremento en los porcentajes de germinación. En ese sentido, los factores CF (Tierra negra y Arena aluvial 2:1+ sin tratamiento pre germinativo) y DF (Tierra de hormiga y Cascara de arroz 3:1+ inmersión en agua a temperatura ambiente 24 horas) presentaron 80 y $73 \%$ respectivamente, bajo la condición ambiental SL, y los factores BG (Arena aluvial y tierra negra proporción 2:1 + inmersión en agua a temperatura ambiente 24 horas), AG (Arena aluvial + inmersión en agua a temperatura ambiente 24 horas) y CF (Tierra negra y arena aluvial proporción 2:1 + sin tratamiento pre germinativo) registraron porcentajes de 60,51 y $51 \%$ respectivamente, bajo la condición ambiental SM (Tabla 2). 
Tabla 2. Número de semillas germinadas y potencia germinativa por factor para cada condición lumínica plena exposición solar (SL) y bajo malla poli-sombra(SM).

\begin{tabular}{l|c|c|c|c}
\hline \multirow{2}{*}{ Factores } & \multicolumn{4}{|c}{ GERMINACIÓN } \\
\cline { 2 - 5 } & N. . SL. & No. SM & \% SL & \% SM \\
\hline AF & 31 & 19 & 69 & 42 \\
\hline AG & 29 & 23 & 64 & 51 \\
\hline BF & 30 & 10 & 67 & 22 \\
\hline BG & 30 & 27 & 67 & 60 \\
\hline CF & 36 & 23 & 80 & 51 \\
\hline CG & 32 & 21 & 71 & 47 \\
\hline DF & 33 & 19 & 73 & 42 \\
\hline DG & 21 & 19 & 47 & 42 \\
\hline EF & 19 & 11 & 42 & 24 \\
\hline EG & 19 & 10 & 42 & 22 \\
\hline Total & 280 & 182 & 62 & 40 \\
\hline
\end{tabular}

\section{Crecimiento en diámetro y altura}

\section{Diámetro}

En cuanto a crecimiento en diámetro los resultados muestran diferencia estadísticamente significativa entre factores, y entre condiciones lumínica, además las interacciones entre factores y condición lumínica también presentaron diferencia significativa, ya que los valores de $(P)$ fueron inferiores al nivel de confianza $(P<0.5)($ Tabla. 3).

Tabla 3. Análisis de varianza (ANOVA) para el crecimiento en diámetro de Cariniana pyriformis

\begin{tabular}{l|c|c|c|c|c}
\hline \multicolumn{5}{c}{ Análisis de la varianza en diámetros por factor } \\
\hline F.v & SC & gl & CM & F & p-valor \\
\hline Modelo. & 4,51 & 19 & 0,24 & 6,82 & $<0,0001$ \\
\hline FACTOR & 2,4 & 1 & 2,4 & 68,78 & $<0,0001$ \\
\hline CONDICIÓN AMBIENTAL & 0,91 & 9 & 0,1 & 2,91 & 0,0038 \\
\hline FACTOR ${ }^{*}$ CONDICIÓN AMBIENTAL & 1,21 & 9 & 0,13 & 3,84 & 0,0003 \\
\hline Error & 4,18 & 120 & 0,03 & & \\
\hline Total & 8,7 & 139 & & & \\
\hline
\end{tabular}


El incremento en diámetro de las plántulas de abarco $C$ pyriformis expuestas en la condición ambiental SL fue en promedio de $0,89 \mathrm{~cm}$, donde los factores con mayores valores fueron DF (Tierra de hormiga + Cascara de arroz 3:1+ Sin tratamiento pre germinativo) y CF (Tierra negra + Arena aluvial 2:1+ Sin tratamiento pre germinativo), mientras que las plántulas evaluadas en la condición ambiental SM registraron valores promedios de $0,63 \mathrm{~cm}$. Los mejores promedios de crecimiento se presentaron en los factores EF (Tierra negra + Arena aluvial + Gallinaza 1:1:1+ sin tratamiento pre germinativo) y EG (Tierra negra + Arena aluvial + Gallinaza 1:1:1+ inmersión en agua a temperatura ambiente 24 horas) (Tabla 4).

Tabla 4. Crecimiento promedio en diámetro de las plántulas de Abarco Cariniana pyriformis por factor y condición ambiental.

\begin{tabular}{|c|c|c|}
\hline Factores & Condición ambiental SL & Condición ambiental SM \\
\hline $\mathrm{AF}$ & 0,93 & 0,55 \\
\hline$A G$ & 0,65 & 0,61 \\
\hline $\mathrm{BF}$ & 0,87 & 0,38 \\
\hline$B G$ & 0,94 & 0,65 \\
\hline $\mathrm{CF}$ & 1,03 & 0,61 \\
\hline CG & 0,71 & 0,71 \\
\hline DF & 1,04 & 0,49 \\
\hline DG & 0,93 & 0,7 \\
\hline $\mathrm{EF}$ & 0,9 & 0,84 \\
\hline$E G$ & 0,91 & 0,76 \\
\hline Promedio & 0,89 & 0,63 \\
\hline
\end{tabular}

\section{Altura}

El crecimiento en altura muestra diferencia estadísticamente significativa entre factores, y entre condiciones lumínica, además las interacciones entre factores y condición lumínica también presentaron diferencia significativa, ya que los valores de $(P)$ fueron inferiores al nivel de confianza $(P<$ 0.5) (Tabla. 5).

Tabla 5. Análisis de varianza (ANOVA) para el crecimiento en altura de las plántulas de abarco Cariniana pyriformis

\begin{tabular}{l|c|c|c|c|c|c}
\hline \multicolumn{7}{c}{ Análisis de la varianza en altura } \\
\hline F.V & SC & gl & CM & F & p-valor \\
\hline Modelo. & 4686,55 & 19 & 247,0 & 3,93 & $<0,0001$ \\
\hline CONDICIÓN AMBIENTAL & 1136,01 & 1 & 1136,0 & 18,12 & $<0,0001$ \\
\hline FACTOR & 2149,93 & 9 & 239,0 & 3,81 & 0,0003 \\
\hline CONDICIÓN AMBIENTALFACTOR.. & 1400,61 & 9 & 156,0 & 2,48 & 0,0123 \\
\hline Error & 7524,34 & 120 & 63,0 & & \\
\hline Total & 12210,9 & 139 & & & \\
\hline
\end{tabular}


El incremento en altura de las plántulas de abarco C. pyriformis expuestas bajo la condición ambiental SL registra promedios de $22,27 \mathrm{~cm}$. Los factores con mejor comportamiento fueron CF (Tierra negra y Arena aluvial 2:1+ Sin tratamiento pre germinativo) y CG (Tierra negra y Arena aluvial 2:1+ Inmersión en agua a temperatura ambiente por 24 horas) para la condición ambiental SL. Por su parte, la condición SM registro promedio $17,64 \mathrm{~cm}$, donde los factores que representaron mejores promedios fueron EF (Tierra negra, Arena aluvial y Gallinaza 1:1:1+Sin tratamiento pre germinativo) y EG (Tierra negra, Arena aluvial y Gallinaza 1:1:1 + Inmersión en agua a temperatura ambiente por 24 horas) (Tabla 6). Por lo tanto, para el abarco C. pyriformis los sustratos tierra negra y arena en presencia de luz, presentan efectos positivos, para el incremento en altura de las plántulas en vivero.

Tabla 6. Crecimiento promedio en altura de las plántulas de abarco Cariniana pyriformis por factor y condición ambiental.

\begin{tabular}{l|c|c}
\hline Factores & $\begin{array}{c}\text { Condición } \\
\text { ambiental SL }\end{array}$ & $\begin{array}{c}\text { Condición } \\
\text { ambiental SM }\end{array}$ \\
\hline AF & 21,21 & 11,36 \\
\hline AG & 9,37 & 15,78 \\
\hline BF & 20,56 & 8,73 \\
\hline BG & 23,9 & 17,93 \\
\hline CF & 28,94 & 17,17 \\
\hline CG & 28,9 & 20,01 \\
\hline DF & 27,79 & 14,61 \\
\hline DG & 23,84 & 21,54 \\
\hline EF & 22,97 & 25,56 \\
\hline EG & 22,36 & 22,49 \\
\hline Promedio & 22,98 & 17,52 \\
\hline
\end{tabular}

\section{Supervivencia}

El porcentaje de supervivencia de las plántulas de C. pyriformis registro un promedio de 98,4 y $92,5 \%$ para la condición SM y SL respectivamente. En consecuencia, la condición ambiental SM, obtuvo el mayor número de factores con registros de supervivencia de $100 \%$, entre los que, se pueden mencionar el AF (Arena aluvial + Sin tratamiento pre-germinativo), BF (Arena aluvial y Tierra negra $2: 1+$ Sin tratamiento pre-germinativo), CF (Tierra negra y Arena aluvial 2:1 + Sin tratamiento pre-germinativo), CG (Tierra negra y Arena aluvial 2:1 + Inmersión en agua a temperatura ambiente por 24 horas), DG (Tierra de hormiga y Cascara de arroz 3:1 + Inmersión en agua a temperatura ambiente por 24 horas), EF (Tierra negra, Arena aluvial, Gallinaza 1:1:1 + Sin tratamiento pre-germinativo) y EG (Tierra negra, Arena aluvial y Gallinaza 1:1:1 + Inmersión en agua a temperatura ambiente por 24 horas), mientras que, en la condición ambiental SL, solo el factor DG (Tierra de hormiga y Cascara de arroz 3:1 + Inmersión en agua a temperatura ambiente por 24 horas).

\section{Discusión}

La germinación es el proceso de recuperación de la actividad biológica en los diferentes tejidos de la semilla, lo cual ocurre mediante las fases de hidratación, germinación y crecimiento dando origen así a una plántula (Pérez, 2003). El tiempo de inicio de la germinación de las semillas de $C$. Pyriformis ocurrió 13 días después de la siembra. Lo cual difiere de lo reportado por Pinilla (2015); Mosquera et al. (2012); Palencia et al. (2006) que obtuvieron germinación a los 15 días después de la fecha de siembra. Por lo tanto, Dalling (2002) indica que las semillas en los bosques húmedos tropicales, es común que germinen en el menor tiempo después de sembradas, por su corta longevidad. Arriaga et al. (1994) mencionan que la poca capacidad germinativa se atribuye a semillas recalcitrante que pierden viabilidad rápidamente. El proceso de germinación de las semillas de $C$. pyriformis se extendió hasta el día 27 después de la fecha siembra. Dato que concuerda con lo reportado por Diez \& Moreno (1998) y difiere de lo reportado por Mosquera et al. (2012); Palencia et al. (2006); CORANTIOQUIA (2007); Pinilla (2015), quienes indican que el proceso de germinación culmina entre los 32 y 43 días después de 
la siembra del material vegetal. Por su parte, CONIF (2004), afirma que las semillas de abarco $C$. pyriformis presentan buenos resultados en el proceso de germinación cuando su contenido de humedad se encuentra alrededor de los $8.15 \%$. Sin embargo, González (1991), plantea que algunas especies arbóreas presentan germinación tardía, debido a muchos factores característicos de la semilla, inmadurez fisiológica del embrión, como también los factores externos como luminosidad, temperatura, humedad, aireación y sustrato.

El tratamiento pre germinativos inmersión en agua ambiente durante 24 horas y el testigo registraron porcentajes de germinación de 33,1\% y $29,1 \%$ respectivamente. Dato que ratifica lo expresado por Triviño \& Torres (2005), quienes sugieren la inmersión de las semillas de abarco $C$. pyriformis en agua ambiente para acelerar el proceso de germinación, mientras CORANTIOQUIA (2007), Betancur \& Raigosa (1973), afirman que, las semillas de $C$. Pyriformis no necesitan tratamiento pre germinativo para su germinación. En ese sentido, Guerra \& Montoya (2013); Mosquera et al. (2012); Pinilla (2014) afirman que, la textura del sustrato es la principal causa de las variaciones en la germinación de las semillas de abarco C. pyriformis, además el exceso de humedad por la poca permeabilidad es un condicionante en el proceso. Por lo tanto, Rivera et al. (2013) afirman que, las plántulas de abarco responden adecuadamente a la condición luz, siempre y cuando estén ubicadas sobre un sustrato rico en nutrientes y buena porosidad. Según Pérez (2003); Garzón, Montenegro \& López (2005); Suarez \& Melgarejo (2010) la humedad, la temperatura, el oxígeno y la iluminación son los factores externos que inciden en la germinación de las semillas. Sin embargo, Aparicio, Cruz \& Alba. (1999) mencionan que son las características físicas, químicas y biológicas de los sustratos los que garantizan la germinación de las semillas y el correcto desarrollo de la plántula.

Las plántulas de C. pyriformis localizadas en la condición SL registraron mejor incremento promedio, en comparación con las situadas en la condición
SM con valores promedios de 0,89 y $0,63 \mathrm{~cm}$ respectivamente (Tabla 4). Por lo tanto, el crecimiento en diámetro de esta especie, está influenciado por la cantidad de luz que recibe en sus primeros estadios de vida, sobre un sustrato rico en nutrientes. Al respecto, Mexal \& Landis (1990), afirman que, el crecimiento en diámetro que experimentan las plántulas influyen en la robustez y supervivencia de las mismas.

En cuanto al incremento en altura, la especie presentó valores promedios de 22, 27 y 17, $64 \mathrm{~cm}$ para plántulas expuestas en las condiciones SL y SM respectivamente. Esto valida lo expuesto por CORANTIOQUIA (2007) quien afirma, que el C. pyriformis pertenece al gremio ecológico semiheliofita (Esta especie se comporta bien tanto en sombra como en luz). Al respecto, Da silva et al. (2007) sugieren que el incremento en altura que experimentan las plantas que se adaptan bajo ambas condiciones es una respuesta morfo genética típica, debido a que desarrollan un mecanismo importante de adaptación. Los tratamientos Tierra negra + arena aluvial proporción 2:1 con valor promedio $28,9 \mathrm{~cm}$ y tierra negra + arena aluvial + gallinaza con valor promedio de $24 \mathrm{~cm}$ en las condiciones SL y SM respectivamente, superan los obtenidos por Mosquera et al. (2012); Pinilla (2015), quienes registraron alturas promedios de 7,9 y $14,9 \mathrm{~cm}$ en los sustratos S4 (Gallinaza y arena aluvial proporción 2:1) y So (Arena aluvial) respectivamente. Por su parte, Negreros, Apodaca \& Cal. (2010) afirma que el potencial de crecimiento de una plántula se relaciona con las características físicas de la misma al momento de sembrarse y que una característica muy importante es el balance entre el tamaño de su sistema radicular y la cantidad follaje. Entre tanto, Prieto, Vera \& Merlin (2003) indican que la expectativa de supervivencia y crecimiento inicial de las plántulas forestales dependen de la capacidad de la misma y de su complejo sistema de condiciones morfológicas, fisiológico y sanitario de la plántula al aplicar las técnicas en el manejo de vivero. Por otra parte, Cetina et al. (2002) afirman que el desarrollo inicial y supervivencia reflejan la calidad de la planta y su capacidad de adaptación a campo. 


\section{Conclusiones}

La especie $C$. pyriformis presenta una germinación epigea, este proceso inicia a los 15 días después de la fecha de siembra y se extiende hasta el día 27. Las semillas de esta especie germinan tanto en condición lumínica como en sombra, este fenómeno indica que estas semillas son no-fotoblásticas, es decir que para su germinación es indiferente la presencia o ausencia de luz.

La interacción de los tratamientos pre germinativos con los sustratos orgánicos genera efectos positivos en el proceso de germinación de las semillas de abarco C. pyriformis, lo anterior está sustentado en el aumento del porcentaje.

Los factores compuestos de sustratos orgánicos tierra negra y arena aluvial proporción 2:1 presentaron los mejores promedios para el crecimiento en diámetro y altura en la condición SL. Mientras que para las mismas variables bajo condición SM se obtuvo mejor comportamiento en los factores constituidos por tierra negra, arena aluvial y gallinaza proporción 1:1:1.

\section{Literatura citada}

1. Aparicio, R., Cruz, J \& Alba, L. (1999). Efecto de seis sustratos sobre la germinación de Pinus patulasch, et chan, Pinus montezumae lamb. y Pinus pseudostrobus lindl. en condiciones de vivero. Rev. Forest Veracruz, 1 (2), 4-31. Veracruz, México: Universidad de Veracruz.

2. Arriaga, M., Cervantes, V. \& Vargas, M. (1994). Manual de reforestación con especies nativas: Colecta y preservación de semillas, propagación y manejo de plántulas. 1 edición. México, D. F. 179 p.: Instituto Nacional de Ecología, SEDESOL, UNAM.

3. Betancur, G. \& Raigosa, J. (1973). Características y propiedades germinativas de las semillas de Abarco (Cariniana Pyriformis Miers). Rev Facultad de Agronomía. 28 (2), 1-4: Universidad de Antioquia

4. Bryant, D. (1997). The Last Frontier Forests. World Resources Institute, Washington, Estados Unidos.

5. Cárdenas, L. \& Salinas, N. (2006). Libro Rojo de plantas de Colombia. Especies maderables amenazadas parte $1.232 \mathrm{p}$.

6. Centro Agronómico Tropical de Investigación y Enseñanza-CATIE, (2002). Planificación del manejo diversificado de bosques latifoliados húmedos tropicales. Turrialba, Costa Rica. 28 p.
7. Cetina, M., González, H., Ortega, D., Vargas, H. \& Villegas, M. (2002). Supervivencia y crecimiento en campo de Pinus greggii engelm. previamente sometido a podas o sequía en vivero. Revista Agrociencia. 36, 233-241: Colegio de posgraduados Mexico.

8. Comisión Nacional Forestal-CONAFOR, (2010). Prácticas de reforestación. Manual básico. Jalisco, México. $56 \mathrm{p}$.

9. Corporación Autónoma Regional del Centro de Antioquia-CORANTIOQUIA. (2007). Manejo de las semillas y la propagación de diez especies forestales del bosque húmedo tropical. Boletín Técnico Biodiversidad $\mathrm{N}^{\circ}$ 3. $64 \mathrm{p}$.

10. Corporación Autónoma Regional para el Desarrollo Sostenible del Chocó-CODECHOCO. (2010). Análisis de resultados estadísticos de movilización, removilización y renovación en el departamento del Chocó. 20 p.

11. Corporación Nacional de Investigación y Fomento Forestal-CONIF. (2004). Programa de investigación en semillas forestales de especies nativas-INSEFOR. Informe final. $33 \mathrm{p}$. Recuperado de http://bibliotecadigital. agronet.gov.co/bitstream/11348/6703/2/016.pdf

12. Dalling, W. (2002). Ecología de las semillas. 12 p. Recuperado de http://academic.uprm.edu/ jchinea/cursos/ecolplt/lectesc/DALLING.PDF

13. Da silva, R., Siebeneicller, J. \& Chagas, J. (2007). Desenvolvimiento inicial de plántulas de Theobroma grandiflorum (Willd, ex spreng) Schum, sobre el influencia de sombreamiento. Acta Amazónica. 37 (3), 365-370.

14. Díaz, M \& Gast, H. (2009). El Chocó Biogeográfico de Colombia: un lugar único en el planeta. $24 \mathrm{p}$.

15. Diez, G. M. \& Moreno, H. F. (1998). Morfología de semillas y plántulas de árboles de los bosques húmedos tropicales del suroriente de Antioquia. Facultad Nacional Agropecuaria, 51 (2), 9-50. Recuperado de file:///C:/Users/casa/Downloads/28916-104090-1PB\%20(1).pdf

16. Farfán, V. (2012). Arboles con potencial para ser incorporados con café. 9 p. Recuperado de http://www.academia.edu/11149747/Arboles_potencial_SAF

17. Federación Nacional de Industriales de la MaderaFEDEMADERAS. (2012). La reforestación en Colombia visión de futuro. 2 p. Recuperado de http:// fedemaderas.org.co/wp-content/uploads/2012/04/ VER-PRESENTACI\%C3\%93N-GREMIAL-Y-SECTORIAL.pdf

18. Galeano, G. E., Calderón, H., Dueñas, I. \& Tobón, H. 2007. Abarco: Cariniana pyriformis Miers. En D. Cárdenas \& N. Salinas (Ed.), Libro rojo de plantas de Colombia. Volumen 4. Especies maderables amenazadas: primera parte. (pp 63-67). Bogotá, Colombia.

19. Garzón, G., Montenegro, R. \& López, B. (2005). Uso de aserrín y acículas como sustrato de germinación y crecimiento de Quercus humbold tii (Roble).Colombia Forestal, 9 (18), 98-108.

20. González, E. (1991). Recolección y germinación de semillas de 26 especies arbóreas del bosque húmedo tropical. Rev Biológica Tropical, 39, 47-51. 
21. Guerra, L. \& Montoya U. (2013). Evaluación de la capacidad de germinación de la semilla del Abarco ( $\mathrm{Ca}$ riniana pyriformis) en la subregion del Urabá. (Tesis de pregrado) Ingenierìa Agroforestal. Universidad Nacional Abierta y a Distancia. Turbo, Colombia. 7 p.

22. Hernández, J., Hurtado, R., Ortiz T. \& Walschburger, T. (1992). La Diversidad Biológica en Iberoamérica. Acta Zoológica Mexicana, 105- 152.

23. Holdridge, L. (1987). Ecología basada en zonas de vida. San José, Costa Rica. 216 p.: Editores Técnicos Asociados.

24. Informe de Cobertura Económica Regional - ICER. (2012). 69 p. Recuperado de www.dane.gov.co/files/ icer/2012/choco_icer_12.pdf

25. Instituto de Investigaciones Ambientales del PacíficoIIAP. (2006). Esquema de Ordenamiento Territorial. Documento de Diagnóstico. 128 P. Quibdó, Colombia: Editores Técnicos Asociados.

26. Klinger, B. (2008). Estado de conservación de las especies forestales amenazadas, Abarco "Cariniana pyriformis", Jigua negro "Ocotea cernua", Guayaquil "Centrolobium paraense", Guayacán amarillo "Tabebuia crysantha" y Pino amarillo "Podocarpus sp." en los municipios chocoanos de Riosucio, Carmen del Darién, Istmina, Río Quito y Juradó. Revista Bioetnia. 137-147.

27. Mexal, J \& Landis T. (1990). Target seedlings concepts: Height and diameter. En: S. J. Campbell, and T. D. Landis (eds.). Target Seedling Symposium: roceedings, Combined Meeting of the Western Forest Nursery Associations. (pp: 17-36).

28. Ministerio de Ambiente, Vivienda y Desarrollo Territorial. (2010). Resolución N³83, 23 de febrero de 2010. 29 p.: Editores Técnicos Asociados.

29. Mosquera, D., Medina H. H. \& Martínez, M. (2012). Germinación y crecimiento inicial del abarco Cariniana pyriformis: Una alternativa para la conservación. Biodiversidad Neotropical, 2 (1), 3-12.

30. Negreros, C., Apodaca, M. \& Carl, W. (2010). Efecto de sustrato y densidad en la calidad de plántulas de Cedro, Caoba y Roble. Madera y Bosques, 16 (2), 7-18.
31. Palencia, C., Gómez, S., Martin, S. \& Guiza P. (2006). Especies forestales para uso en sistemas agroforestales con cacao: una alternativa para el occidente de Boyacá. 11 p. Boyacá, Colombia.

32. Pérez, F. (2003). Germinación y dormición de semillas. En A. Sánchez., M. Arroyo., \& R. M. Navarro. (Eds.). Material vegetal de reproducción: manejo, conservación y tratamiento. (117 - 200 p). Barcelona, España.

33. Pinilla C. (2014). En prensa. Propagación de la especie forestal abarco (cariniana pyriformis) en el corregimiento de Salero, Chocó. 3 p. Unión Panamericana, Colombia.

34. Prieto, R., Vera, C. \& Merlín, E. (2003). Factores que influyen en la calidad de brízales y criterios para su evaluación en vivero. Durango: Instituto Nacional de Investigaciones Forestales, Agrícolas y Pecuarias. $14 \mathrm{p}$.

35. Rivera, M., Peñuela, M., Jiménez., R. \& Vargas, J. (2013). Ecología y Silvicultura de Especies Útiles Amazónicas: Abarco (Cariniana micrantha Ducke), Quinilla (Manilkara bidentata (A. DC.) A. Chev.) y Violeta (Peltogyne paniculata Benth.). 181 p. Recuperado de http:// www.bdigital.unal.edu.co/36632/6/9789587616347.pdf

36. Secretaria de Convenio sobre la Diversidad Biológica. (2009). Informe sobre la conservación de las especies vegetales: Una revisión de los progresos realizados en la aplicación de la estrategia mundial para la conservación (GSPC). 7 p.

37. Suárez, D., \& Melgarejo, L. (2010). Biología y germinación de semillas. En L. M. Melgarejo (Ed.). Experimentos en fisiología vegetal (pp.13-249). Bogotá, Colombia: Universidad Nacional de Colombia.

38. Triviño, D. T. \& Torres, R. F. (2005). Manual práctico manejo de semillas y viveros agroforestales. $41 \mathrm{p}$. Recuperado de http://www.franciscotorres.co/wp-content/ uploads/2013/01/manual-semillas-y-viveros.pdf

39. Unidad Internacional de Conservación NacionalUICN, (2012). Categorías y Criterios de la Lista Roja de la UICN: Versión 3.1. Segunda edición. Gland, Suiza y Cambridge, Reino Unido: UICN. 34 pp.
Conflicto de Intereses

Los autores declaran no tener ningún conflicto de intereses

Recibido: 11 de marzo de 2016 Aceptado: 19 de abril de 2016 